\title{
Different configurations of 25-order structure of Steiner triple systems of counting
}

\author{
Wei Tian ${ }^{1, a}$, Xiaoyi $\mathrm{Li}^{2, \mathrm{~b}}$, Wanxi Chou ${ }^{3, \mathrm{c}}$ \\ 1. School of Mathematics and Systems Science, Shenyang normal University, Liaoning Shenyang, \\ 110034, China.
}

2. School of Civil Engineering and Architectures, Anhui University of Sciences and Technology, Anhui Huainan,232001, China.

E-mail: 18840607717@163.com

Keywords: Triple System; Complete trimap; Count

\begin{abstract}
A method of constructing and technology view for sub graph decomposition of $K_{v}$ is proposed.,and it clarifies the application completely trimap and complete bipartite graph in combination Design; Order to clarify the structure of the basic ideas of Steiner triple systems 25 Order introduced the whole process of construction Steiner triple systems and computing.The conclusion is: the text of the constructors and counting methods Steiner triple systems are effective and replicable.
\end{abstract}

\section{Introduction}

Block design theory is an important branch of the combinatorial mathematics and plays an important role in experiment design, competition arrangement, digital communication areas. In 1850 a British mathematician Thomas P.Kirkman posed a famous 15 schoolgirls problem and solved this problem in the same year [1-5]. In 1971 D.R. Ray-Chaudhari and R.M. Wilson published a paper with topic Solution of Kirkman's schoolgirls problem to show how to construct Kirkman triple systems of order $6 n+3$ [6-10]. In 1961 a Chinese mathematician Lu Jiaxi posed the decomposable condition of BIBD design [11-12]. It has been an open problem for over hundred years to determine whether there exists a Kirkman triple system of order $6 n+3$ for each $n=0,1,2,3$. In 2003 a method of constructing Kirkman triple systems of order $s \times t$ by using main matrix and subsidiary matrix is proposed by the author of this paper, and Kirkman triple systems of order $v=27,45,81,135$, are constructed [13-15], the open problem mentioned above can be solved hopefully[5].

\section{Basic theory}

Definition 1: Supposing Top Set $V(G)=\left\{c_{1}, c_{2}, \cdots, c_{v}\right\}$, Edge Set

$E(G)=\left\{c_{1} c_{2}, c_{1} c_{3}, \cdots, c_{1} c_{v}, c_{2} c_{3}, \cdots, c_{2} c_{v}, \cdots, c_{v-1} c_{v}\right\}$ and $G$ is complete to $K_{v}$, if we put $|E(G)|=v(v-1) / 2$ sides into triangular matrix so that it can exist the relation with any edge $c_{i} c_{j}$, top $c_{i}$ and top $c_{j}$, then this triangular matrix called side matrix, and referred to as $K_{v}^{\prime}$.

Definition 2: Supposing Top Set is $V(G)=\left\{c_{1}, c_{2}, \cdots, c_{v}\right\}$ and $V(G)$ include $V_{i}=\left\{c_{m}, c_{m+1}, \cdots, c_{m+t-1}\right\}, V_{j}=\left\{c_{p}, c_{p+1}, \cdots, c_{p+t-1}\right\}, V_{k}=\left\{c_{q}, c_{q+1}, \cdots, c_{q+t-1}\right\}$ three subsets, if each top of $V_{i}$ neighbor top $t$ of $V_{j}$ and top $t$ of $V_{k}$, then graph $G$ called complete trimap and denoted $K_{t, t, t}^{(i, j, k)}$, which three sub-graph is called complete ipartite graph, respectively $K_{t, t}^{(i, j)}$, $K_{t, t}^{(i, k)}, \quad K_{t, t}^{(j, k)}$, meanwhile, $K_{t, t, t}^{(i, j, k)}=K_{t, t}^{(i, j)} \cup K_{t, t}^{(i, k)} \cup K_{t, t}^{(j, k)}$

If $t \times t$ complete graph $K_{3}$ exist in the complete trimap $K_{t, t, t}^{(i, j, k)}$ has separated, then $t \times t$ triple system matrix $K_{t, t, t}^{(i, j, k)}$ can be delieved as 


$$
K_{t, t, t}^{(i, j, k)}=\left\{\begin{array}{cccc}
\{m, p, q\} & \{m, p+1, q\} & \cdots & \{m, p+t-1, q+t-1\} \\
\{m+1, p+1, q\} & \{m+1, p+2, q\} & \cdots & \{m+1, p, q+t-1\} \\
\vdots & \vdots & \vdots & \vdots \\
\{m+t, p+t-1, q\} & \{m+t-1, p, q\} & \cdots & \{m+t-1, p+t-2, q+t-1\}
\end{array}\right\}
$$

Apparently, we put the fixed of minimum number from $m, p, q, V_{i}, V_{j}, V_{k}$ into matrix $K_{t, t, t}^{(i, j, k)}$, then we can obtain $t \times t$ complete graph $K_{3}$, but $K_{t, t, t}^{(i, j, k)}$ has $t$ kinds of structure rather than only one.

Theorem 1:: Supposing Top Set is $V(G)=\left\{C_{1}, C_{2}, \cdots, C_{v}\right\}$ and $|V(G)|=v=s \times t-s+1$, s, $t$ already exists Steiner triple's order,then it must be exist $s \times t-s+1$ order of steiner triple and this steiner triple's structure is equivalent to $v(v-1) / 6$ kinds decompose from a complete graph $K_{v}$.

The specific steps of constructing the Steiner triple system with the order of $s \times t-s+1$ are that:

(1)The edge matrix $K_{v}{ }^{\prime}$ can be divided as $t$ edge matrices $K_{t}^{(1)}, K_{t}^{(2)}, \cdots, K_{t}^{(s)}$ of complete graphs $K_{v}^{\prime}$, and $s(s-1) / 2$ edge matrices of $K_{t-1, t-1}^{(i, j)}, K_{t-1, t-1}^{(i, k)}, \ldots \ldots, K_{t-1, t-1}^{(j, k)}$ with complete bipartite graph., then we get the edge matrix $K_{v}^{1 A}$.

(2)Let s complete graphs $K_{t}^{(1)}, K_{t}^{(2)}, \cdots, K_{t}^{(s)}$ of $K_{v}^{\prime}$ with the order of $t$ be devided into $t(t-1) / 2$ complete graphs $K_{3}$, and $s(s-1) / 2$ complete bipartite graphs $K_{t-1, t-1}^{(i, j)} K_{t-1, t-1}^{(i, k)} K_{t-1, t-1}^{(j, k)}$ can be merged as $s(s-1) / 2$ triple systems matrix $K_{t-1, t-1, t-1}^{(i, j, k}$, which be constituted with $(t-1)(t-1)$ complete graphs $K_{3}$.

\section{2.method of construction Steiner triple systems of order 25}

If edge matrix $K_{25}^{\prime}$ of complete graphs $K_{25}$ can be divided into 3 complete graphs $K_{9}^{(1)}, K_{9}^{(2)}, K_{9}^{(3)}$ with order 9 of edge matrix $K_{9}^{1(1)}, K_{9}^{1(2)}, K_{9}^{1(3)}$,and edge matrix $K_{8,8}^{(1,2)}, K_{8,8}^{(1,3)}, K_{8,8}^{(2,3)}$ of 3 complete bipartite graphs $K_{8,8}^{(1,2)}, K_{8,8}^{(1,3)}, K_{8,8}^{(2,3)}$.

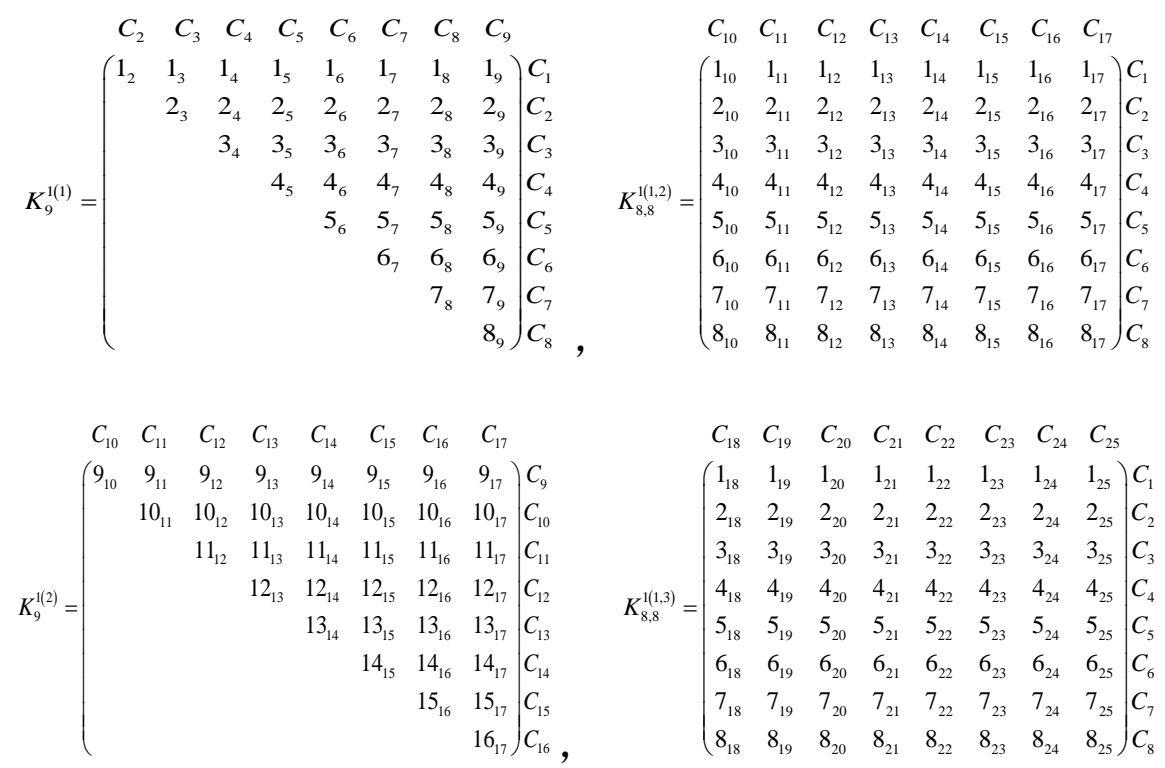




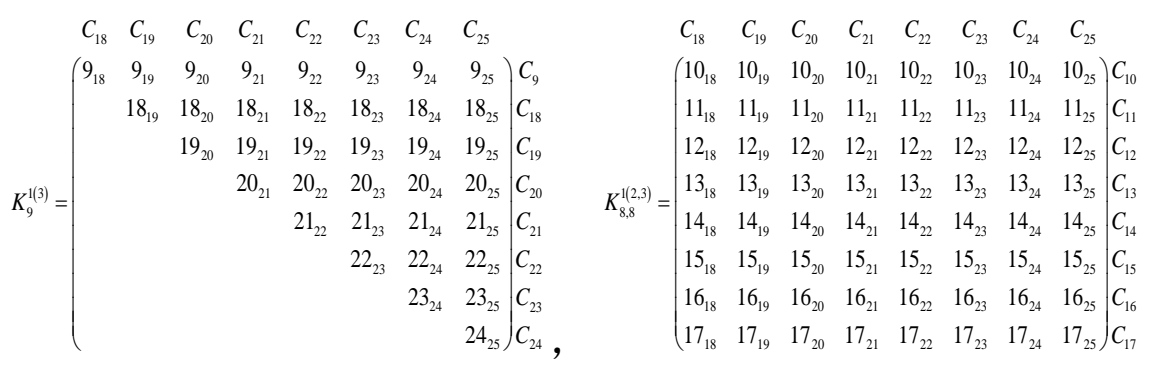

Then edge matrix $K_{9}^{1(1)}, K_{9}^{1(2)}, K_{9}^{1(3)}$ of 3 complete graphs can be divided into $9(9-1) / 6$ complete graphs $K_{3}$,according to Steiner triple systems $S T_{1}(9), S T_{2}(9), \cdots S T_{9}(9)$ of order 9 , and 3 complete bipartite graphs $K_{8,8}^{(1,2)}, K_{8,8}^{(1,3)}, K_{8,8}^{(2,3)}$ can be merged as Steiner triple systems matrix $K_{8,8,8}^{(1,2,3)}$ of $8 \times 8$ complete graphs $K_{3}$. Finally, $9(9-1) / 2$ complete bipartite graphs $K_{3}$ and matrix $K_{8,8,8}^{(1,2,3)}$ constructed Steiner triple systems $S T_{1}(25)$ of order 25.Supposing the division of $9(9-1) / 6$ complete graphs $K_{3}$ of $K_{9}^{(1)}, K_{9}^{(2)}, K_{9}^{(3)}$ with $S T_{2}(9), S T_{3}(9), \cdots, S T_{7}(9)$ respectively,and division of proposal $8 \times 8$ complete graphs $K_{3}$ of complete trimp graphs $K_{8,8,8}^{(1,2,3)}$ should be transformed accordingly,so we get some different configurations triple systems of Steiner triple systems $S T_{2}(25), S T_{3}(25), \cdots, S T_{7}(25)$ of order 25.

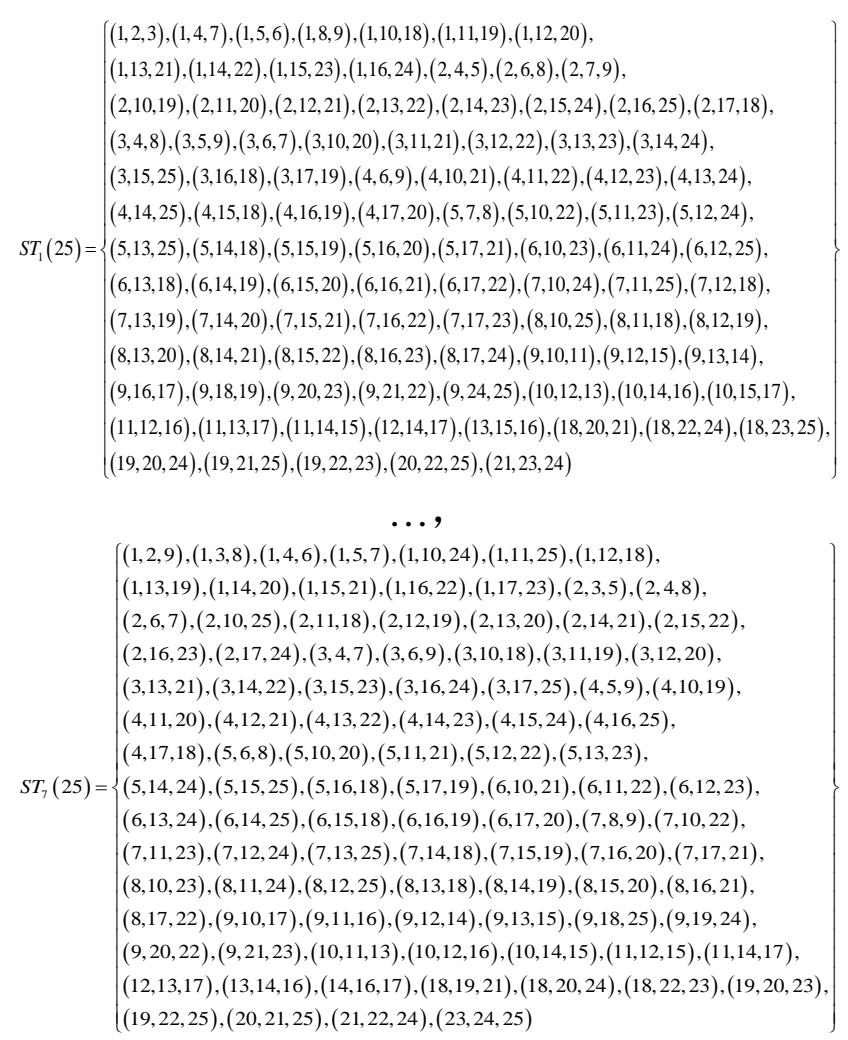

\section{Counting of Steiner triple system with 25 order}

The number of Steiner triple systems with 25 order depended on division proposal number $N^{(1)}$ of $9(9-1) / 6$ complete graphs $K_{3}$ of complete graphs $K_{9}^{(i)}$ with order 9,coming from matrix $K_{25}^{\prime}$ of complete graphs $K_{25}$ with order 25 , and division proposal number $N^{(2)}$ of $8 \times 8$ complete graphs $K_{3}$, and $N^{(1)}$ decideed number of Steiner triple systems with order 9.Construction result and enumeration of 105 Steiner triple systems with order 9 be proved, and $N^{(1)}=105, N^{(2)}=8$. Obviously.According to Multiplication principle,number of 25 Order Steiner triple systems are that: $N=105 \times 8$. 


\section{REFERENCES}

[1] J.H. van Lint, R.M.Wilson.A Course in Combinatiorics [M]. Beijing: china Machine Press.2004.

[2] Fred S.Roberts, Barry Tesman.Applied Combinatorics [M].Beijing: China Machine Press.2007.

[3] Douglas B.West. Introduction to Graph Theory [m]. Beijing: China Machine Press.2004.

[4] L.R.Foulds.Graph Theory Applications [M].New Youk: Springer-Verlag.1992.

[5] J. C. Bermond, An application of the solution of Kirkman's schoolgirl problem: The decomposition of the symmetric oriented complete graph into 3-circuits, Discrete Mathematics, Vol. 8, No. 4, 301-304, 1974.

[6] M. J. Grannell, T.S. Griggs, K.A.S. Quinn, Smallest defining sets of directed triple systems, Discrete Mathematics, Vol. 309, No. 14, 4810-4818, 2009.

[7] D. M. Deng, R. Rolf, and S. Hao, on the existence and application of incomplete nearly Kirkman triple systems with a hole of size 6 or 12, Discrete Mathematics, Vol.261, No.1-3,209-233, 2003.

[8] D. M. Deng, R. Rolf, and S. Hao, Further results on nearly Kirkman triple systems with subsystems, Discrete Mathematics, Vol. 270, No. 1-3, 99-114, 2003.

[9] J. X. Yin, C. M. Wang, Kirkman covering designs with even-sized holes, Discrete Mathematics, Vol. 309, No. 6, 1422-1434, 2009.

[10] L. Ji, A new existence proof for large sets of disjoint Steiner triple systems, Journal of Combinatorial Theory, Series A, Vol. 112, No. 2, 308-327, 2005.

[11] H. Peter, on the chromatic number of Steiner triple systems of order 25, Discrete Mathematics, Vol. 299, No. 1-3, 120-128, 2005.

[12] B. Darryn, M. Barbara, and Q. Kathleen, S. Bridget, Existence and embeddings of partial Steiner triple systems of order ten with cubic leaves, Discrete Mathematics, Vol. 284, No. 1-3, 83-95, 2004. [13] K. Daniel, M. Edita, P. Attila, J.S. Sereni, Characterization of affine Steiner triple systems and Hall triple systems, Electronic Notes in Discrete Mathematics, Vol. 29, 17-21, 2007.

[14] A. D. Forbes, M. J. Grannell, and T. S. Griggs, On 6-sparse Steiner triple systems, Journal of Combinatorial Theory, Series A, Vol. 114, No. 2, 235-252, 2007.

[15] K. Petteri, R. J. Patric, ÖstergÅrd, Svetlana Topalova, Rosen Zlatarski, Steiner triple systems of order 19 and 21 with subsystems of order 7, Discrete Mathematics, Vol. 308, No. 13, 2732-2741, 2008.

[16] X. Y. Li, Z. D. Xu, and W. X. Chou, A method of constructing Kirkman triple system of higher order, in proceeding of Control and Decision Conference (CCDC), 2318-2322, 2010.

[17] X. Y. Li, Z. D. Xu, and W. X. Chou, A new method of constructing Steiner triple systems, in proceeding of Control and Decision Conference (CCDC), 3760-3764, 2010.

[18] H. Cao, Y. Tang, On Kirkman packing designs KPD (\{3,4\},v), Discrete Mathematics, Vol. 279, No. 1-3, 121-133, 2004.

[19] L.J. Ji, J.G. Lei, Further results on large sets of Kirkman triple systems, Discrete Mathematics, Vol. 308, No. 20, 4643-4652, 2008.

[20] H. Peter, on the chromatic number of Steiner triple systems of order 25, Discrete Mathematics, Vol. 299, No. 1-3, 120-128, 2005.

[21] CHOU Wan-xi. A Method of Constructing Kirkman Triple System of Order $k \times t$ [J]. Mathematics In Practice and Theory, 2004, 34(9):144-150.

[22] CHOU Wan-xi. Seiner Triple System and Its Construction Met [J]. Journal of Anhui University of Science and Technology(Natural Science), 2004, 24(3):76-80.

[23] Ai Mingyao, Zhang Runchu. UNIFORMAITY OF BLOCK DESIGNS [J]. Journal of Nankai University (Natural Science), 2003, 36(2):89-92.

[24] LIU Jian-guo, GUO Qiang, XIA Zun-quan. Optimal Block Designs in Mixed Effects Models [J]. Mathematics in Practice and Theory, 2005, 35(5):97-103.

[25] HAN Gang, LI Hui-ling. Block designs admitting an automorphism group with an alternating socle[J]. Journal of Zhejiang University (Sciences Edition), 2003, 29(3):241-245. 